\title{
Excitation dependence of resonance line self-broadening at different atomic densities
}

\author{
Hebin Li, ${ }^{1}$ Vladimir A. Sautenkov, ${ }^{1,2}$ Yuri V. Rostovtsev, ${ }^{1}$ and Marlan O. Scully ${ }^{1,3}$ \\ 1 Department of Physics and Institute for Quantum Studies, \\ Texas AEM University, College Station, Texas 77843-4242 \\ 2 P.N. Lebedev Institute of Physics, Moscow 119991, Russia \\ 3 Princeton Inst. for the Science and Technology of Materials and \\ Dept. of Mech. \& Aerospace Eng., Princeton University, 08544
}

(Dated: November 6, 2018)

\begin{abstract}
We study the dipole-dipole spectral broadening of a resonance line at high atomic densities when the self-broadening dominates. The selective reflection spectrum of a weak probe beam from the interface of the cell window and rubidium vapor are recorded in the presence of a far-detuned pump beam. The excitation due to the pump reduces the self-broadening. We found that the selfbroadening reduction dependence on the pump power is atomic density independent. These results provide experimental evidence for the disordered exciton based theory of self-broadening, and can be useful for the description of the interaction of a strong optical field with a dense resonance medium.
\end{abstract}

\section{INTRODUCTION}

For many applications and fundamental physics it is necessary to know the non-linear optical response of a resonance atomic gas under conditions when dipole-dipole interactions between atoms in the ground and excited states can not be neglected.

There was a common opinion that calculations of selfbroadening can be performed by using the two-particle approximation in the fast collision limit (impact collisions) as well as in the opposite limit, static interactions [1, 2, 3, 4]. In Ref. [4], the theory of self-broadening is developed on the basis of disordered exciton in a dense resonance medium, in which many particle interaction should be taken into account. By using this model it was shown that the self-broadening is a combination of collision and static atomic interactions. A ratio of the static width to the collision width is independent on atomic density in a wide range where thermal motion of atoms can be neglected.

It has been shown that the probing of the homogeneous and inhomogeneous contributions to the linewidth can be performed efficiently by nonlinear optical methods such as photon echoes and hole burning [5, 6]. The inhomogeneous component of the spectral line could be sensitive to the optical saturation. Recently by using time resolved femto-second spectroscopy, the non-Markovian collision dynamics and the bi-exponentional correlation of energy level fluctuations has been observed in a dense potassium vapor and simulations of molecular dynamic are in good agreement with experimental results 7]. The slow exponential component is attributed to long-range resonant attraction in a dense atomic vapor. By using CW pump-probe technique, the excitation dependence of the self-broadening is observed in rubidium [8, 9] and potassium [10, 11] vapors. Nevertheless, note that in these papers the measurements have been performed only at selected atomic densities.

In the current paper the selective reflection spectrum from the interface between the cell window and rubidium vapor are recorded in the presence of a far-detuned pump beam. We have studied the excitation dependence of selfbroadening of a resonance atomic line at different atomic densities in the range where self-broadening of atomic line is stronger than Doppler broadening. The excitation dependence of self-broadening is found to be independent on the atomic density. These results support the disorder exciton based theory of self-broadening [4].

\section{EXPERIMENT}

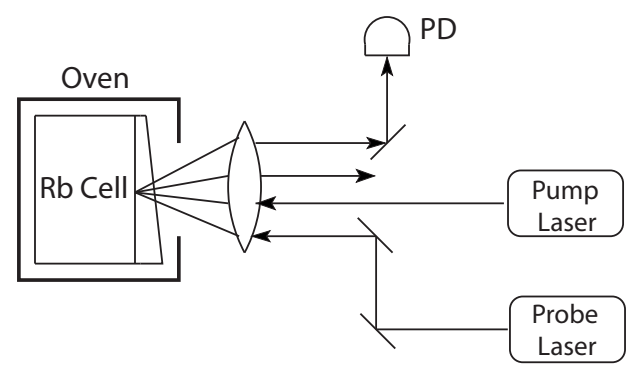

FIG. 1: The experimental schematic. Pump and probe laser beams are focused on the interface between rubidium vapor and window. The reflection is recorded by a photodetector.

The experiment was performed with a pump-probe scheme shown in Fig. 1, The reflectivity and frequencymodulated (FM) reflectivity spectra of a dielectric-vapor (rubidium) interface were measured at the $5^{2} S_{1 / 2} \rightarrow$ $5^{2} P_{3 / 2}$ transition $\left(D_{2}\right.$ line, shown in Fig. 2) of rubidium atoms. Please note that the excited state hyperfine structure is not resolved in our experiment since the splittings are less than the Doppler width $(0.5 \mathrm{GHz})$ at room temperature. The probe laser is a free running diode laser (linewidth $20 \mathrm{MHz}$ ) which can be scanned over $30 \mathrm{GHz}$ around $D_{2}$ transition. The frequency of the probe laser is calibrated by reference to the absorption of a rubidium 
cell at room temperature. The power of the probe beam is small enough (less than $100 \mu \mathrm{W}$ ) such that no saturation effects need to be considered. The pump laser (an extended cavity diode laser) provides a laser beam with power up to $180 \mathrm{~mW}$, and it is far-tuned $(20 \mathrm{GHz})$ to the red wing of $D_{2}$ line to avoid coherent effects. The frequency of the pump laser is determined by beating with the probe laser. Both pump and probe beams are focused down to a spot with $100 \mu \mathrm{m}$ diameter by a $15 \mathrm{~cm}$ lens, and they are overlapped at the inner surface of the cell window.

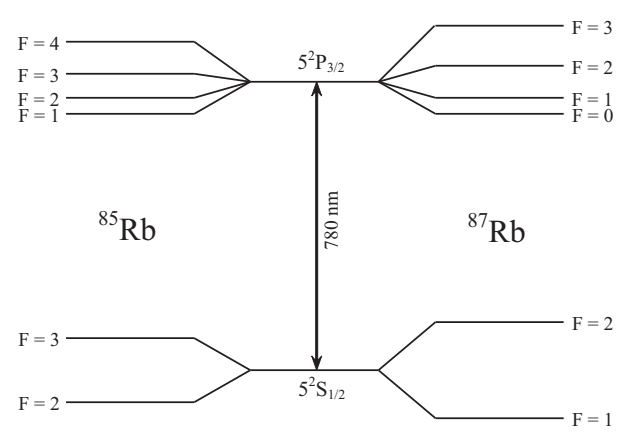

FIG. 2: The energy diagram of the $D_{2}$ line of ${ }^{85} \mathrm{Rb}$ and ${ }^{87} \mathrm{Rb}$. The hyperfine splittings of the excited states are less than the Doppler width $(0.5 \mathrm{GHz})$ at room temperature and are not resolved in our experiment.

The cell contains natural abundance of rubidium vapor. The cell is made of sapphire, and the windows are Garnet crystal which is free of birefringence. The cell can be heated to reach a high atomic density $\left(\mathrm{N} \approx 10^{17}\right.$ $\mathrm{cm}^{-3}$ ). The windows are slightly wedged in order to separate the reflections from two surfaces.

The reflected beam from the interface between the rubidium vapor and the window is sent to a photodetector (PD). The signal from the photodetector is processed by a lock-in amplifier while we frequency modulate the probe laser with modulation depth of $37 \mathrm{MHz}$ at frequency of 8 $\mathrm{kHz}$. FM reflectivity spectra is used to improve the signal to noise ratio in our experiment, and it can reveal subtle details of change in reflectivity. A typical FM reflectivity spectrum is shown in Fig. 3 as curve (a) which was obtained at the atomic density $\mathrm{N}=1.3 \times 10^{17} \mathrm{~cm}^{-3}$. The dipole-dipole interaction and collision broadening dominate at this atomic density, the spectral width due to self-broadening is larger than the ground state hyperfine splitting and the ground state hyperfine structures in reflectivity spectra that can be seen at low atomic density are not resolved. While we apply the pump laser beam, atoms are partially excited and the dipole-dipole interaction is reduced. Thus, the self-broadened line width is also reduced [8, 9, 10, 11], and the ground state hyperfine structures start to be revealed as described in [9, 10]. The narrowed FM spectrum with pump power $\mathrm{P}=180 \mathrm{~mW}$ is shown as curve (b) in Fig. 3 ,

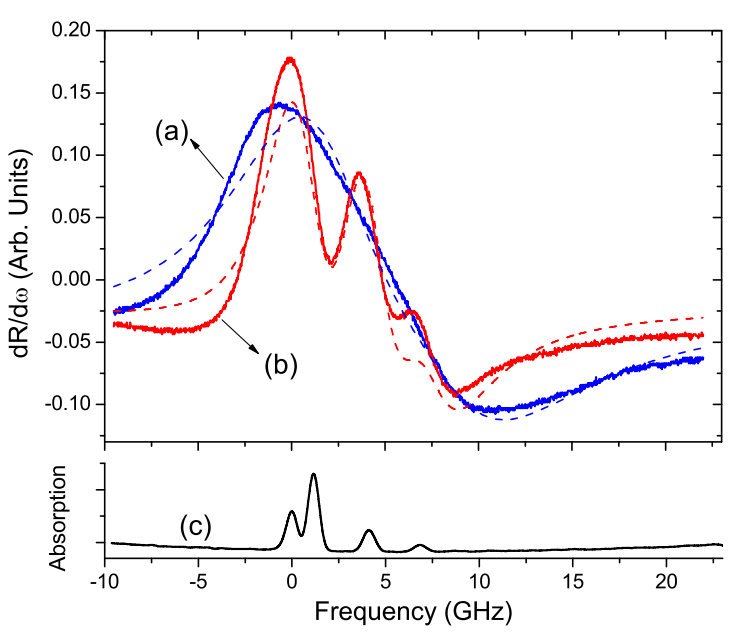

FIG. 3: (Color online) FM reflectivity spectra at atomic density $\mathrm{N}=1.3 \times 10^{17} \mathrm{~cm}^{-3}$. Blue (a) and red (b) solid curves correspond to the cases without pump laser and with pump laser $(\mathrm{P}=180 \mathrm{~mW})$, respectively. The dashed curves are corresponding fitting results. Curve (c) is the absorption spectra of a reference $\mathrm{Rb}$ cell, where the ground state hyperfine splitting is resolved.

As described in [10], the reflection spectra as well as the FM reflection spectra can be interpreted in terms of the dielectric coefficient of atomic vapor. Taking into account the excitation, the dielectric coefficient $\epsilon$ of a two-level atomic system is given as

$$
\epsilon(\omega)=1+\frac{k \eta N}{\Delta \omega+\Delta \Omega-i \Gamma},
$$

where $N$ is the atomic density, $\Delta \omega$ is the frequency detuning, $\Delta \Omega$ is the overall line shift which includes Lorentz and non-Lorentz shift [12, 13, 14], and $\Gamma$ is the self-broadened linewidth. The constant $k$ is given by $k=f c r_{e} \lambda$, where $f$ the oscillator strength of transition, $r_{e}$ the classical radius of electron, $\lambda$ the wavelength of transition, and $c$ is the speed of light in vacuum. An excitation factor $\eta$ is defined as the fractional population difference between ground and excited states

$$
\eta=\frac{N_{g}-N_{e} g_{g} / g_{e}}{N}
$$

where $N_{g}$ and $N_{e}$ are the ground and excited state atomic densities respectively, $g_{g}$ and $g_{e}$ are the degeneracies of the ground and excited states respectively. Maximum excitation corresponds to $\eta=0$ and zero excitation $\eta=$ 1. Using this expression for the dielectric coefficient $\epsilon$ and Fresnel formula, we are able to calculate the reflectivity and FM spectra which is the derivative of reflectivity with respect to frequency. 
In order to obtain the width and excitation factor from the experimental data, we use the expression of FM spectra to fit the experiment data by leaving the self-broadened width $\Gamma$, the excitation factor $\eta$ and the line shift $\Delta \Omega$ as fitting parameters. The excitation factor $\eta$ is normalized to unity for the case where no pump laser is applied. In our experiment, $\mathrm{Rb}$ vapor contains natural abundance of ${ }^{85} \mathrm{Rb}$ and ${ }^{87} \mathrm{Rb}$ which gives rise to four doppler-broadened absorption lines in the absorption spectra. All of four components are taken into account in the fitting of reflection spectra. Each component is given a normalized oscillator strength. The dashed curves in Fig. 3 are the examples of the fitting. For the case without the pump laser, the fitted width is $13.0 \pm 0.3$ $\mathrm{GHz}$ and $\eta=1.0$; for the case with the pump laser (laser power $\mathrm{P}=180 \mathrm{~mW}$ ), the fitted width is $4.98 \pm 0.05 \mathrm{GHz}$ and $\eta=0.36$. At the same atomic density $\left(\mathrm{N}=1.3 \times 10^{17}\right.$ $\mathrm{cm}^{-3}$ ), the FM spectrum is recorded when we apply the pump laser with different powers. The fitting of these FM spectra gives the widths corresponding to the different excitation factors. The fitting results are shown as the red squares in Fig. 4, where the width is plotted as a function of the excitation factor $\eta$. The dashed line in Fig. 4 is a linear fit $(y=a+b x$, where the dependent variable $x$ represents the excitation $\eta$ and $a$ and $b$ are fitting parameters) of the excitation dependence of the width at atomic density $\mathrm{N}=1.3 \times 10^{17} \mathrm{~cm}^{-3}$, and the slope (fitting parameter $b$ ) is $12.7 \mathrm{GHz}$.

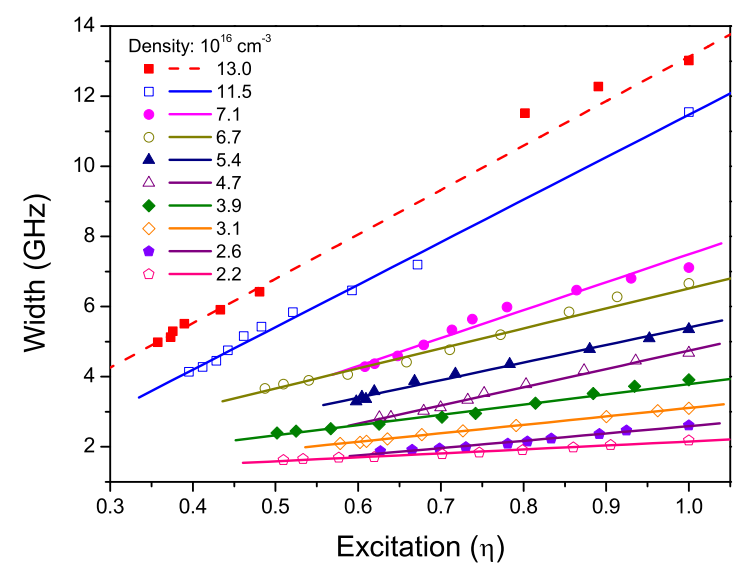

FIG. 4: (Color online) The fitting value of the width is plotted as a function of the excitation factor $\eta$. Different colors represent the results at different atomic density from $\mathrm{N}=1.3 \times 10^{17}$ $\mathrm{cm}^{-3}$ to $\mathrm{N}=2.2 \times 10^{16} \mathrm{~cm}^{-3}$. The straight lines are the linear fits.

We determine the widths and excitation factors by measuring and fitting the FM reflection spectra at different atomic densities from $\mathrm{N}=1.3 \times 10^{17} \mathrm{~cm}^{-3}$ to $\mathrm{N}=2.2 \times 10^{16} \mathrm{~cm}^{-3}$. The measured density dependence of the self-broadened rubidium $\mathrm{D}_{2}$ linewidth is the same as in Refs. [15]. The excitation dependence of the width for different atomic densities is shown in Fig. 4 with different colors. The corresponding linear fit gives the slope (width $/ \eta$ ) for each density. In Fig. [5, the slope is plotted as a function of the atomic density. The solid line is a linear fit. If the slope for each density is normalized by taking the ratio of the slope to the width without the pump laser at each density, the normalized slopes are close to unity. As shown in Fig. 6, the value of the normalized slope is $0.90 \pm 0.05$. According to our simple model for the fit, the normalized slope is density independent. It indicates that the self-broadening at high atomic density is a combination of collision and static spectral broadening (inhomogeneous profile).

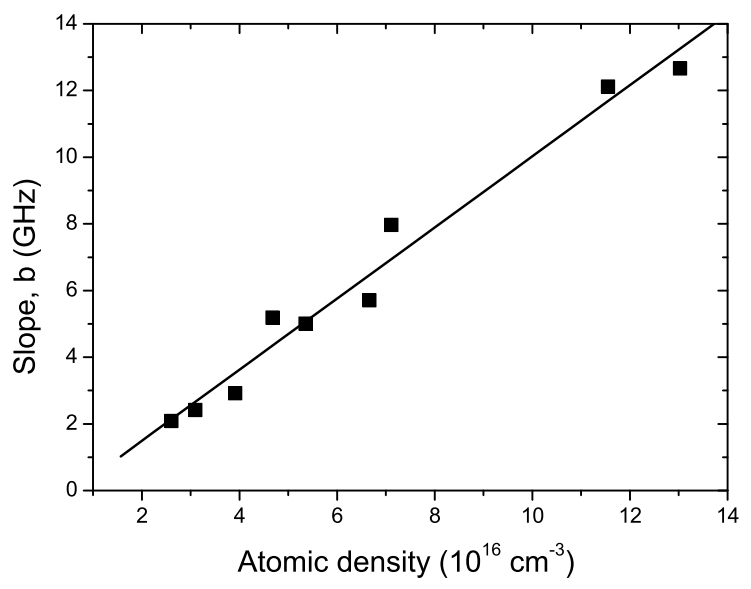

FIG. 5: The slope of the width dependence on the atomic density is plotted as a function of the atomic density. Squares are the results of fitting from Fig. 4 The solid straight line is a linear fit.

\section{CONCLUSION}

We have observed that the excitation dependence of self-broadening is the same in the range of atomic density from $2.2 \times 10^{16}$ to $1.3 \times 10^{17} \mathrm{~cm}^{-3}$, where dipole-dipole interactions are a dominant source of spectral broadening. Our results support the predictions of the theoretical model developed in Ref. [4]. The obtained results can be useful for understanding of excitation processes in a condensed media such as solutions, glasses, polymers, proteins and molecular crystals. In atomic gases it will be interesting to study a possible transition from many body interactions (disordered excitons) [4, 7, 8] to the resonance two-body interaction (impact collisions) at lower atomic densities [1, 2]. Probably the studies will 
EEC-0540832 (MIRTHE ERC).

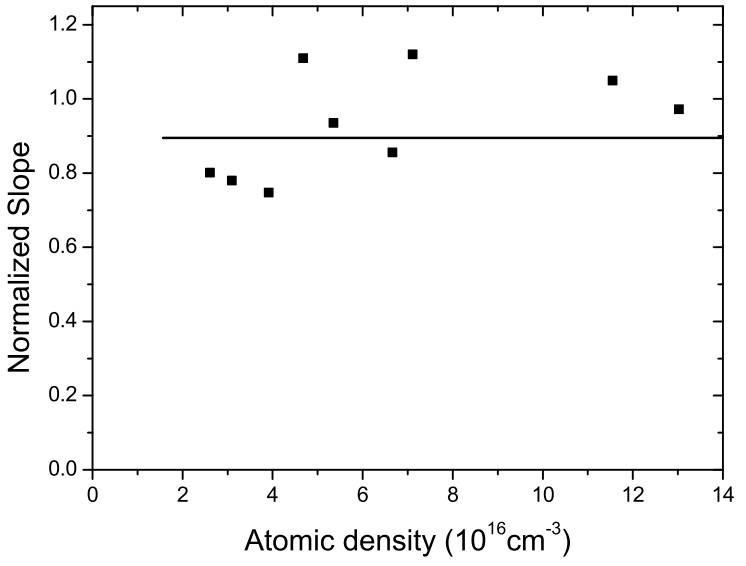

FIG. 6: Normalized slope is plotted as a function of the atomic density. Squares are the data extracted from experimental results. The solid line is the linear fit with a zero slope. The fit value of the normalized slope is $0.90 \pm 0.05$.

require applications of non-linear optical methods in frequency and time domain as the complimentary spectroscopic techniques. We shall note that additional information about the dipole-dipole interaction in a dense gas may be obtained by using a nano-cell [16].

\section{ACKNOWLEDGEMENT}

We thank S.T. Cundiff, S. Mukamel, D. Sarkisyan and R.W. Welch for useful discussions. Also, we wish to acknowledge D. Sarkisyan and T. Varzhapetyan for the high-temperature cell. This work is supported by the Welch Foundation (Grant A-1261) and the NSF grant
[1] E. L. Lewis, Phys. Rep. 58, 1 (1980).

[2] Yu. Vdovin and N.A. Dobrodeev, Sov. Phys. JETP. 18, 544 (1969).

[3] M. Movre, G. Pichler, J. Phys. B 13, 697 (1980); V. Horvatic, M. Movre, R. Beuc and C. Vadla, ibid 26, 3679 (1993).

[4] J.A. Leegwater and S. Mukamel, Phys. Rev. A 49, 146 (1994).

[5] S. Mukamel, Principles of nonlinear optical spectroscopy, (Oxford University Press, New York, 1995).

[6] Y.J. Yan and S. Mukamel, J. Chem. Phys. 94, 179 (1991).

[7] V. O. Lorenz and S. T. Cundiff, Phys. Rev. Lett. 95, 163601 (2005); V. O. Lorenz, S. Mukamel, W. Zhuang, and S. T. Cundiff, Phys. Rev. Lett. 100, 013603 (2008).

[8] V. A. Sautenkov, H. van Kampen, E. R. Eliel, and J. P. Woerdman, Phys. Rev. Lett. 77, 3327 (1996).

[9] H. Li, T.S. Varzhapetyan, V. A. Sautenkov, Y.V. Rostovtsev, H. Chen, D. Sarkisyan, M.O. Scully, Appl. Phys. B 91, 229 (2008).

[10] H. van Kampen, V. A. Sautenkov, C. J. C. Smeets, E. R. Eliel and J.P. Woerdman, Phys. Rev. A 59, 271 (1999).

[11] V. A. Sautenkov, Y. V. Rostovtsev, E. R. Eliel, Phys. Rev. A 78, 013802 (2008).

[12] R. Friedberg, S. R. Hartmann and J. T. Manassah, Phys. Rep. 7, 101 (1973); Phys. Rev. A 42, 5573 (1990).

[13] J. T. Manassah, Phys. Rep. 101, 359 (1983).

[14] J. J. Maki, M. S. Malcuit, J. E. Sipe, and R. W. Boyd, Phys. Rev. Lett. 67, 972 (1991); J. J. Maki, W. V. Davis, R. W. Boyd, and J. E. Sipe, Phys. Rev. A 46, 7155 (1992).

[15] K. Niemax, M. Movre, and G. Pichler, J. Phys. B 12, 3503 (1979); R. Kondo, S. Tojo, T. Fujimoto, and M. Hasuo, Phys. Rev. A 73, 062504 (2006).

[16] T. Varzhapetyan1, A. Nersisyan1, V. Babushkin1, D. Sarkisyan1, S. Vdović and G. Pichler, J. Phys. B 41, 185004 (2008). 